Available online on 15.09.2019 at http://jddtonline.info
$\begin{aligned} & \text { C } 2011-18, \text { publisher and licensee JDDT, This is an Open Access article which permits } \\ & \text { unrestricted non-commercial use, provided the original work is properly cited }\end{aligned}$

Open $\overbrace{\text { Access }}$

Research Article

\title{
Formulation-Development and In-Vitro-In Vivo Evaluation of Gastroretentive Floating Tablet Incorporating Clarithromycin
}

\author{
Gautam D. Mehetre*1, Dubey A ${ }^{2}$ \\ ${ }^{1}$ Department of Pharmaceutics, Dr. Rajendra Gode College of Pharmacy, Malkapur, Dist- Buldana, 443101 Maharashtra, India \\ 2 Department of Pharmaceutical Science, Chatrapati Shahu Ji Maharaj University, Kanpur, India
}

\begin{abstract}
The objective of the work is to summarize the applicability, manufacturing possibilities, excipients and the types of floating drug delivery systems and to optimize a floating, mucoadhesive system using Clarithromycin as the drug aiming at the eradication of Helicobacter pylori having desired floating and drug release properties based on preliminary excipient examination. Direct compressed (DC) tablet was chosen as dosage form being a cost-effective technology for pharmaceutical industry requiring fewer procedures. Before the implementation of the pharmaceutical technological aims, analysis of critical factors influencing the manufacture was carried out. Reproducible manufacturing processes are required to achieve suitability and tablets uniformity to achieve the uniform properties of tablets, which could influence experimental parameters. Ishikawa diagram evaluation was created, which is a commonly used graphical method to identify factors resulting in an overall effect on product design and quality imperfection. The aim was to reveal affecting factors on uniformity of DC tablets in order to standardize all possible conditions and adjustments. Critical factors are indicated separately in particular method sections.
\end{abstract}

Keywords: H. pylori, Clarithromycin, Floating Tablets, In Vitro Evaluation, In Vivo Evaluation.

Article Info: Received 23 June 2019; $\quad$ Review Completed 11 Aug 2019; Accepted 19 Aug 2019; Available online 15 Sep 2019

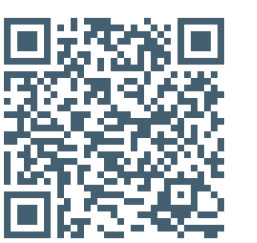

Cite this article as:

Mehetre GD, Dubey A, Formulation-Development and In-Vitro-In Vivo Evaluation of Gastroretentive Floating Tablet Incorporating Clarithromycin, Journal of Drug Delivery and Therapeutics. 2019; 9(5):67-81

http://dx.doi.org/10.22270/jddt.v9i5.3559

Prof. G.D. Mehetre. Asst. Prof., Department of Pharmaceutics, Dr. Rajendra Gode College of Pharmacy, Malkapur, Dist- Buldhana, Pin code 443101, Maharashtra, India

\section{INTRODUCTION:}

The most frequent application of medicines is the peroral way of administration, which provides easy to take option, relatively low therapeutic cost, various formulations and applicable technologies 1 . Its spread is shown by the fact that more than $50 \%$ of commercially available medicines are orally applied preparations 2 . Higher patient compliance may be experienced due to their easy application. Although among the per os administered preparations, few are designed with biopharmaceutical aspect meeting with the physiological environment of the dosage forms. While until the 90's not much, however nowadays more frequently modified drug delivery systems are designed containing special excipients and/or manufactured with special technological methods 1 . With novel preparations having controlled release, patient compliance can be increased more, namely multiple daily administrations can be reduced to once a day administration. Another advantage can be a local drug delivery, with which not only the administration of the medicine can be improved, but also the site-specific efficiency of a particular applied active pharmaceutical ingredient (API) may be optimized.

Based on the Dévay's proposal biopharmaceutical classification system of pharmaceutical preparations, the following classes of drug delivery systems can be distinguished ${ }^{1}$ :

1.1 Time controlled systems based on the effect of time after their administration and the time interval of effect can be the following:

1.1.1 Rapid (e.g. solutions, effervescent preparations, fast dissolving or disintegrating tablets),

1.1.2 Sustained (e.g. extended tablets or tablet implants), 
1.1.3 Delayed (e.g. enteric coated tablets) and,

1.1.4 Pulsatile drug delivery (e.g. repeated bursts of API dissolution) preparations.

1.2 Site-controlled systems, which can be:

1.2.1 Approaches with direct administration of medicine (e.g. directly into muscle or joint) to the target organ or,

1.2.2 Passive and active targeted drug delivery systems:

1.2.3 Passive targeting: nanotechnological drug delivery systems, which are based on accumulation of the drug in the areas around the tumor due to EPR (enhanced permeability and retention) effect 3 ,

1.2.4 Active targeting: nanotechnological drug delivery systems, which are able to identify target cells (e.g. tumor cells) and to bound and penetrate into them in order to achieve specific effect ${ }^{3}$,

1.3 New types of preparations, i.e. site- and timecontrolled systems, the purpose of Combination of the preiviously listed two main classes.

The modification of drug release is always performed to achieve a particular therapeutic aim, with which optimized bioavailability of API(s) can be reached by taking the physiological environment into consideration. In the cases of APIs with short elimination half-life, long acting preparations can be designed with the prolongation of API release and absorption. On the other hand, some acute or emergency cases require the possibility of the most rapid effect of the API, which can be developed by the fast API release from preparations.

Modified drug delivery systems (MDDSs) can be classified based on the time and location of drug release. With per os administered medicines, the location of drug release in the gastrointestinal tract (GIT) may be in: the mouth (e.g. orodisperse, sublingual, buccal DDSs), the stomach (e.g. floating, expandable DDSs), the small intestine and/or the colon (e.g. intestinosolvent, enterosolvent, colon targeted DDSs). Thus the location of drug release can be controlled with an appropriate modification of the preparation, with which sitecontrolled systems can be achieved. During drug release in the oral cavity or in the stomach, not only systemic but also local effects may be taken into consideration, while drug release in the small intestine may be expected to be predominantly systemic. In colon-specific therapy, mostly local effects may develop, since absorption is limited/ minimal. Those modified drug delivery systems, in which the modification is aimed at prolonging the residence time (GRT), are termed gastroretentive drug delivery systems (GRDDS). Via the modification of the time spent in stomach, site- and time-controlled systems may be achieved. Based on the applied technology, gastroretentive systems can be classified into four separate groups explained as under:

1.4 Expandable drug delivery systems hinder their transfer through the pylorus with their expansion, swelling via their size without causing gastric obstruction 4,5 .

1.5 High density drug delivery systems involve formulations of dosage forms having higher average density, than physiological stomach content. Application of high density ingredients are required to use such as barium sulfate $\left(4.50 \mathrm{~g} / \mathrm{cm}^{3}\right)$, zinc oxide $\left(5.61 \mathrm{~g} / \mathrm{cm}^{3}\right)$, titanium dioxide $\left(4.23 \mathrm{~g} / \mathrm{cm}^{3}\right)$. For significant prolongation of GRT, $2.5 \mathrm{~g} / \mathrm{cm}^{3}$ average density is necessary 6 .

1.6 Floating drug delivery systems (FDDS) are those preparations, which are capable for buoyancy on the surface of gastric medium after a particular time. The mechanism of flotation depends on the applied technology. During flotation, preparations have bulk density lower than the gastric fluid $\left(\rho<1.00 \mathrm{~g} / \mathrm{cm}^{3}\right)$ and can remain buoyant without influencing gastric emptying rate. This results in the prolongation of gastric residence time and better control on drug release.

1.7 Mucoadhesive drug delivery systems are capable for bioadhesion onto gastric mucosa resulting in sustaining of GRT, which may cause enhancement of drug absorption in a site-specific manner. Special polymers having mucoadhesive ability are indispensable to apply in these systems, which can adhere to the epithelial surface of the stomach 7. Mucoadhesion may be an approach, which can be combined with former mentioned technologies in order to achieve not only physically but also chemically resulted gastric retention.

1.8 Rationale behind selection of Clarithromycin as suitable drug

Clarithromycin is rapidly and well absorbed from the gastrointestinal tract - primarily in the jejunum - but undergoes extensive first-pass metabolism after oral administration. The absolute bioavailability of a $250-\mathrm{mg}$ clarithromycin tablet is approximately $50 \%$. This spatial absorption window of clarithromycin makes it a suitable candidate to develop it as a floating drug delivery system. Clarithromycin is rapidly and extensively metabolized in the liver. It has elimination half life of about 2-4 hours, which adds to its suitability for development as gastroretentive floating drug delivery system.

\section{MATERIALS AND METHODS}

Paracetamol is used as API in preliminary studies, then followed by clarithromycin as the main drug; Hydroxy propyl cellulose grade B1 and 11, sodium alginate as gelling agent, sodium bicarbonate as gas generating agent, barium sulfate as X-Ray contrast material, Methanol for clarithromycin assay by high performance liquid chromatography (HPLC), potassium dihydrogen phosphate for clarithromycin assay by HPLC, KrebsHensley buffer containing D-glucose, magnesium sulfate, potassium phosphate monobasic, potassium chloride and sodium chloride for ex vivo mucoadhesion studies.

Preliminary - and optimization experiments have been carried out. Principal aim of the research work was the optimization of floating drug delivery system, but the preliminary project and its conclusions were needed to begin designing of optimization project. The Method section contains all method descriptions, which were used either during preliminary or optimization studies.

\subsection{Preformulation methods}

Particle size and shape parameter measurements were done with microscopic examination with the use of $160 \mathrm{x}$ and $640 \mathrm{x}$ magnifications (Zeiss, Axio Imager A1 Microscope, Germany) based on 50 largest separated particles. 
Sphericity $(\Psi)$ was calculated with the following formula:

$$
\text { UI }=4 \pi \frac{\Delta f}{P \frac{2}{L}}
$$

Sphericity of particles describes the form of region on the bases of their circularity. Numerical range is from 0 to 1 . The value of the sphericity for a perfect round shape particle is 1 . Filled area $(A f)$ is the region including any holes on it. Crofton perimeter $(P c r)$ determines circular region with correction optimized for circular objects. Zeiss Axio Vision Rel. 4.7 software (Carl Zeiss, Germany) was used for digital photo analysis.

\subsection{Flowability and density}

Flow properties of L-HPC 11 and B1 were examined to highlight further physical differences. Determination of angle of repose was performed according to 2.9.16. Test of Ph. Eur. Ed. 5.0. Measurements were carried out in triplicate. Angle of repose was calculated. Apparent density examination was carried out by a volumetric device (Erweka SVM 121, Germany) according to 2.9.15. Ph. Eur. 5.0. Bulk densities ( $\rho b u l k)$ were recorded after filling $100.0 \mathrm{~g}$ material into graduated cylinder; tapped densities ( $\rho$ tapped) were recorded after 1250 taps. Using these measurements Carr's indices (Ci) ${ }^{9}$ was calculated. Hausner's ratios $\mathrm{Hr}$ ) were also determined by using the ratios between tapped and bulk density of powders.

\subsection{Wettability}

Force tensiometer (KSV, Sigma 701) was applied to measure water uptake of the L-HPC types. Glass sample holder vessel was used with $1.15 \mathrm{~mm}$ width and with $1.00 \mathrm{~mm}$ diameter glass filter at the bottom holding 50.0 $\mathrm{mg}$ of samples. The materials were immersed into distilled water and $0.1 \mathrm{M}$ hydrochloric acid for 60 minutes respectively. Sampling was done in every 5 seconds. Accuracy of force tensiometer instrument was $0.01 \mathrm{mg}$. Wettability tests were performed in triplicate.

\subsection{Rheological behavior of sodium alginate}

Viscosity grade of sodium alginate was examined with the use of rotational viscometer (Anton PaarRheolab QC, Austria) with standard measuring system (CC27). Temperature dependence of viscosity and flow curves of $1.0 \%$ sodium alginate solution were also determined at $20,25,30,35,37^{\circ} \mathrm{C}$. Viscosity measurements were performed with 100 1/s constant shear rate. Rheological (flow curve) behavior studies were carried out with linear increase of shear rate from 10 to 500 1/s. Three parallel samples were examined. Flow behavior indices $(z)$ of $1 \%$ sodium alginate solutions at different temperature were also calculated based on the Ostwaldde Waele power law model ${ }^{10}$.

\subsection{Drug-excipients interaction studies}

\section{Differential Thermal Analysis (DTA)}

Differential thermal analyzer (Shimadzu DTA-50, Japan) was applied for thermal analysis of Clarithromycin and Clarithromycin-excipient blends. Clarithromycin and excipients were analyzed separately as well as blends with drug-excipient ratios according to the optimized composition (MF_OPT). $10.0 \mathrm{mg}$ of individual samples and blends were scanned in the temperature range of 25$400{ }^{\circ} \mathrm{C}$ under air atmosphere. Temperature rate was 5 ${ }^{\circ} \mathrm{C} / \mathrm{min}$. Peak shifting and melting point were evaluated on thermo grams in order to detect interaction between Clarithromycin and excipients. Three parallel examinations were carried out.

\section{EXPERIMENTAL DESIGN, STATISTICAL ANALYSIS AND OPTIMIZATION}

Several statistical approaches of experimental design originate from the work of R. A. Fischer. In the early $20^{\text {th }}$ century, he showed how important it is to appropriately consider the design and the execution of experiments before they are actually performed to prevent frequently encountered problems.

Design of experiments (DOE) belongs to the field of applied statistics, which deals with planning, analyzing and interpreting controlled studies. Afterwards the data have been gained from experiments they are objectively evaluated in order to understand the mathematical relation between factors (independent variables) and responses (dependent variables). With the application of DOE, fewer experiments are enough to explore their correlation.

The experimental design contains its settings, sequence and is created with the choice of layout type before beginning the studies. Choice of used layout depends on the experimental aim(s). One of the most important aim of DOE is the optimization and assessment of the influence of factors on responses. After objective evaluation of result data, optimization criteria have to be determined based on the desirable responses.

In this work, Design Expert 7.0.0 software was used in order to create the experimental design and response surface plots. Data obtained from the floating tablet properties were analyzed with this software, too. Polynomial models were generated for all responses including linear, quadratic as well as interaction terms. The best model was chosen based on the particular statistical parameters involving coefficient of variation $(\mathrm{CV})$, regression coefficient $(R 2)$ and p-value. The following mathematical equation form was used to evaluate numerical effect of independent variables on responses:

$$
\begin{aligned}
& Y=b_{0}+b_{1} X_{1}+b_{2} X_{2}+b_{3} X_{3}+b_{12} X_{1} X_{2}+b_{13} X_{1} X_{3}+b_{23} X_{2} X_{3}+b_{11} X_{1}^{2}+ \\
& b_{22} X_{2}^{2}+b_{33} X_{3}^{2},
\end{aligned}
$$

Where $Y$ is the response variable, $b 0$ is the intercept, $b i$ is the estimated coefficient of factors. $X 1, X 2$ and $X 3$ are the main effects representing how responses change, when an individual factor changes. Interaction term (X1X2) shows the effect of simultaneous change of factors on responses. $X i^{2}$ is the quadratic effect for evaluation of non-linear correlations.

In this work, two experimental designs were applied.

3.1 The preliminary study focused on the influence of LHPC 11, B1 and their 1:1 mixture on certain properties of sodium alginate based floating drug delivery systems. In this project, face centered central composite design $(\alpha=1)$ was applied with two numerical factors $(X 1, X 2)$ and with three-levels $(+1,0,-1)$. One categorical factor was used involving the types of two L-HPCs and 1:1 mixture of L-HPCs. The two numerical independent variables were the sodium alginate $(X 1)$ and particular LHPC type (X2). 
Factors mean the concentrations (\%) of the materials in the floating tablets. All tablets contained $150 \mathrm{mg}$ paracetamol and fixed amount of excipients contributing effervescent effect and tablet compressibility.
Experimental layout is shown in (Table 1). Dependent variables were the following: floating time, floating lag time, floating force, swelling capability and drug dissolution.

Table 1 Experimental layout of preliminary project

\begin{tabular}{|c|c|c|c|c|c|c|c|c|}
\hline $\begin{array}{l}\text { Exp. } \\
\text { No. }\end{array}$ & $\begin{array}{c}\text { Sodium } \\
\text { alginate, } \\
\text { X1 (\%) }\end{array}$ & $\begin{array}{c}\text { L-HPC } \\
11, X 2 \\
(\%)\end{array}$ & $\begin{array}{c}\text { Exp. } \\
\text { No. }\end{array}$ & $\begin{array}{c}\text { Sodium } \\
\text { alginate, } \\
\text { X1 (\%) }\end{array}$ & $\begin{array}{c}\text { L-HPC } \\
\text { B1, } X 2 \\
(\%)\end{array}$ & $\begin{array}{c}\text { Exp. } \\
\text { No. }\end{array}$ & $\begin{array}{c}\text { Sodium } \\
\text { alginate, } \\
\text { X1 (\%) }\end{array}$ & $\begin{array}{c}\text { L-HPC } \\
\text { 11:B1, } X 2 \\
\text { (\%) }\end{array}$ \\
\hline PFS01 & 0.50 & 0.50 & PFS10 & 0.50 & 0.50 & PFS19 & 0.50 & 0.50 \\
\hline PFS02 & 35.15 & 0.50 & PFS11 & 35.15 & 0.50 & PFS20 & 35.15 & 0.50 \\
\hline PFS03 & 0.50 & 25.00 & PFS12 & 0.50 & 25.00 & PFS21 & 0.50 & 25.00 \\
\hline PFS04 & 35.15 & 25.00 & PFS13 & 35.15 & 25.00 & PFS22 & 35.15 & 25.00 \\
\hline PFS05 & 0.50 & 12.75 & PFS14 & 0.50 & 12.75 & PFS23 & 0.50 & 12.75 \\
\hline PFS06 & 35.15 & 12.75 & PFS15 & 35.15 & 12.75 & PFS24 & 35.15 & 12.75 \\
\hline PFS07 & 17.82 & 0.50 & PFS16 & 17.82 & 0.50 & PFS25 & 17.82 & 0.50 \\
\hline PFS08 & 17.82 & 25.00 & PFS17 & 17.82 & 25.00 & PFS26 & 17.82 & 25.00 \\
\hline PFS09 & 17.82 & 12.75 & PFS18 & 17.82 & 12.75 & PFS27 & 17.82 & 12.75 \\
\hline
\end{tabular}

3.2 For optimization of sodium alginate based floating tablets, face-centered central composite design $(\alpha=1)$ was used with three factors: sodium alginate (X1), L-HPC B1 (X2) and sodium bicarbonate (X3). Each factor was examined in three levels $(+1,0,-1)$. Each tablets contained $250 \mathrm{mg}$ Clarithromycin and constant quantities of excipients contributing effervescent effect and tablet compressibility. Factors mean the concentrations of the materials in the floating tablets. Experimental layout is shown in (Table 2). Dependent variables were the following: floating lag time, maximal floating force, maximal floating force calculated to 100 mg tablet mass, time needed for maximal floating force and drug dissolution.

Table 2 Experimental layout of optimization project

\begin{tabular}{|c|c|c|c|c|}
\hline Exp. No. & $\begin{array}{c}\text { Sodium alginate, } \\
X 1(\%)\end{array}$ & $\begin{array}{c}\text { L-HPC B1, } \\
X 2(\%)\end{array}$ & $\begin{array}{c}\text { NaHC03, } \\
X 3(\%)\end{array}$ & $\begin{array}{c}\text { Total tablet weight } \\
(\mathrm{mg})\end{array}$ \\
\hline MF01 & 5.00 & 30.00 & 8.00 & 463.82 \\
\hline MF02 & 15.00 & 30.00 & 8.00 & 569.48 \\
\hline MF03 & 5.00 & 45.00 & 8.00 & 642.67 \\
\hline MF04 & 15.00 & 45.00 & 8.00 & 865.05 \\
\hline MF05 & 5.00 & 30.00 & 13.00 & 511.25 \\
\hline MF06 & 15.00 & 30.00 & 13.00 & 642.67 \\
\hline MF07 & 5.00 & 45.00 & 13.00 & 737.46 \\
\hline MF08 & 15.00 & 45.00 & 13.00 & 569.03 \\
\hline MF09 & 5.00 & 37.50 & 10.50 & 737.46 \\
\hline MF10 & 15.00 & 37.50 & 10.50 & 538.79 \\
\hline MF11 & 10.00 & 30.00 & 10.50 & 796.18 \\
\hline MF12 & 10.00 & 45.00 & 10.50 & 603.86 \\
\hline MF13 & 10.00 & 37.50 & 8.00 & 686.81 \\
\hline MF14 & 10.00 & 37.50 & 13.00 & 642.67 \\
\hline MF15 & 10.00 & 37.50 & 10.50 & \\
\hline
\end{tabular}

\subsection{Formulation studies}

\subsubsection{Preparation of effervescent floating tablets}

Ingredients of tablets were accurately measured with analytical balance (Kern, ABJ 220-4M, Germany). Powder blends were mixed every time after adding next substance for 3 minutes with the use of mortar and pestle, and finally all the blends were mixed for 10 minutes. The flow properties of blends were qualified to be suitable for direct compression. Eccentric singlepunch tablet press was used using $8,10,12 \mathrm{~mm}$ round concave punches. Compression forces at all batches were adjusted to achieve $50 \pm 5 \mathrm{~N}$ tablet hardness. Tableting was performed and stored at $50 \pm 10 \%$ relative humidity $(R H)$, at $25 \pm 5^{\circ} \mathrm{C}$.

\subsubsection{Studies of floating behavior}

Floating lag time (tlag) is the period from the immersion of the tablet until its buoyancy. Experiments were carried out in $450 \mathrm{ml} 0.1 \mathrm{M}$ hydrochloric acid at $37 \pm 0.5$ ${ }^{\circ} \mathrm{C}$. Durations of floating lag time were visually recorded by camcorder (DCR-SX85E, Sony, Japan). Each test was carried out for 4 hours in triplicate. The process of floating lag time measurements is shown in below figure. 


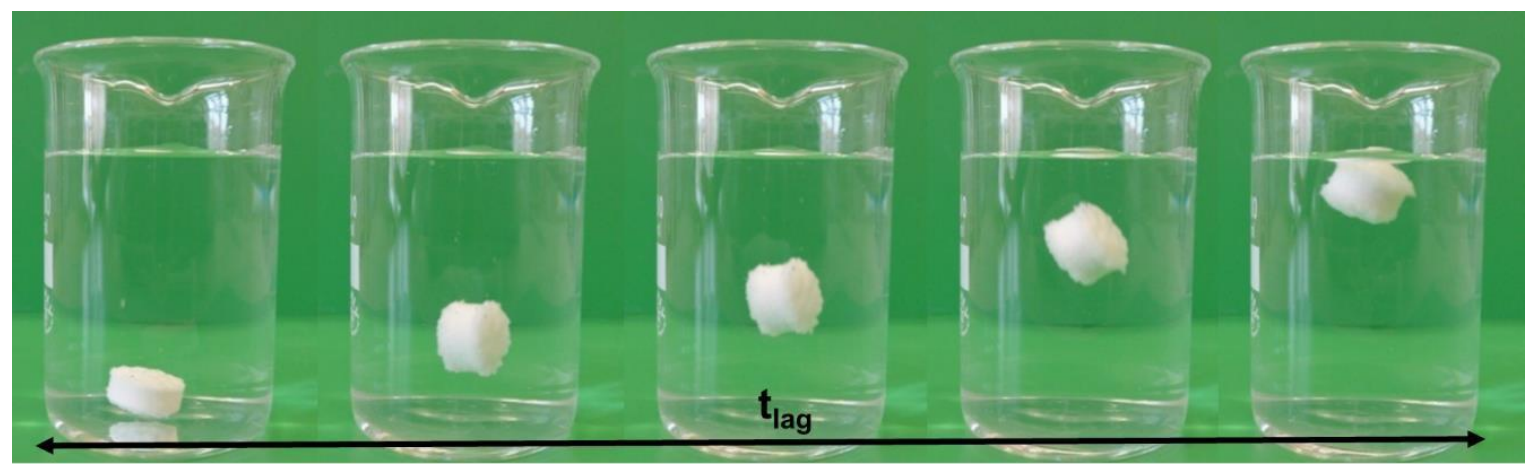

Fig. 1 The processes of floating lag time measurements

\subsubsection{Floating force study}

Floating force measurements were carried out based on the theoretical base described by Timmermans and Moes. 11, 12 KSV Sigma force tensiometer (KSV Instruments Ltd, Helsinki, Finland) was used with $0.1 \mathrm{mg}$ accuracy. Tablets tested in a standard vessel containing $450 \mathrm{ml} 0.1 \mathrm{M} \mathrm{HCl}$, in which a special filtering plate with 2 $\mathrm{mm}$ aperture size was applied. Majority of developing $\checkmark$ Maximal floating force (Fmax),

$\checkmark$ Time ( $t$ Fmax) needed for maximal floating force,

$\checkmark \quad$ Time $(t F 1 / 2)$ required for $50 \%$ of maximal floating force,

$\checkmark \quad$ Maximal floating force calculated for $100 \mathrm{mg}$ tablet mass (Fmax/100mg).

Floating forces were calculated based on the formula described by Cromer 13:

$$
F_{\text {float }}=F_{0}-F_{t}=F_{0}-m_{t} * g=F_{0}-\left(F_{0}+m_{i t} * g\right)
$$

In the equation, Ffloat is the floating force expressed vertically upward by a floating tablet. $F 0$ equals with the multiplication of filtering plate mass in medium with gravitational acceleration $(g)$, which was constantly $39.84 \mathrm{mN}$. $m t$ is the weight measured by the devices, when a tablet pushed the plate upward. $m t$ is the negative weight gradient caused by the tablet directly, which was calculated by subtraction of $m t$ from $\mathrm{mo}$. In absolute value, $m t$ is the weight expressed by the buoyancy of floating tablets. All experiments were performed in triplicate.

\subsubsection{Determination of swelling capability}

Swelling capacities of floating tablets were measured based on the method described by Dorozyn ski et al. 14 . Tablets were weighed (W1), then immersed into glass beaker filled with $200 \mathrm{ml}$ of $0.1 \mathrm{M}$ hydrochloric acid at $37 \pm 0.5{ }^{\circ} \mathrm{C}$. At time $30,60,120,180$ and 240 minutes, tablets were removed from the beaker. After wiping the excess liquid from surface, tablets were reweighed (W2). Swelling index (Si) was calculated.

\subsubsection{Drug release studies}

In vitro dissolution studies of all floating tablets were performed according to $\mathrm{Ph}$. Eur. 2.9.3 dissolution test with paddle apparatus. Stirring speed was 50 RPM (Erweka DT-700, Germany), the medium was $900 \mathrm{ml} 0.1$ $\mathrm{M} \mathrm{HCl}$ tempered to $37 \pm 0.5{ }^{\circ} \mathrm{C}$. Dissolution tests for preliminary project were done for 4 hours, for optimization project were performed for 6 hours. During the studies, $2.5 \mathrm{ml}$ samples were taken at 10, 15, 20, 30, carbon dioxide bubbles passed through the filter plate resulting less noise. Media were exposed with ultrasound to avoid gas formation on filtering plate. During the test, the weight of the filtering plate was continuously measured. Floating tablets pushed the filtering plate vertically upward; hence change of the weight could be registered as a function of time. Evaluated parameters were the following:

45, 60, 90, 120, 180, 240, 300 and 360 minutes. Each sample was filtered through PTFE membrane. All measurements were done in triplicate.

Active substance contents of samples were determined with spectrophotometric method (Jasco V-670, Japan) at their absorption maximum (paracetamol, $\lambda p_{\max }=243$ $\mathrm{nm}$; Clarithromycin, $\lambda^{m} \max =277 \mathrm{~nm}$ ). Linear calibration curve was previously created; all samples were measured within this concentration interval.

In optimization project, dissolution studies of two commercially available Clarithromycin tablets (Klion ${ }^{\circledR}$ $250 \mathrm{mg}$ and, Supplin ${ }^{\circledR} 250 \mathrm{mg}$ ) were also performed for comparison with optimized floating tablets.

\subsubsection{Kinetics of drug release}

Model dependent evaluations of dissolutions were carried out with four mathematical models. Zero order -, first order -, Higuchi - and Weibull kinetic models were used to describe the drug release from floating tablets 15 . Fundamentals of first order kinetics were first described by Noyes-Whitney equation which was later modified several times by Brunner et al. 16, then Hixson and Crowell. The model can be applied in dosage forms such as porous matrices containing water-soluble drugs 17 . The first model Higuchi's kinetics for planar homogeneous matrix system was used accordingly 18 . Weibull's model is a commonly used model, which fits various kinds of dissolution profiles ${ }^{19}$. During evaluation linear transformation was carried out on each dissolution profile and the equation of the fitted linear line was determined according to the following formula:

$$
Q_{t}=s t+n_{\text {diss }}
$$

Where $Q t$ is the drug dissolved in time $t, s$ is the slope of the line, ndiss is y-intercept.

\subsubsection{Ex vivo mucoadhesion studies}

Two most frequently performed mucoadhesion studies were done: the detachment force and rheological mucoadhesion measurements. The former was performed with rat gastric mucosa, the latter one with extracted rat gastric mucus. 
Wistar rats (250-350 g) were bred in a temperaturecontrolled room having a $12 \mathrm{~h}$ light/dark cycle, provided with standard rodent chow and water ad libitum. For harvesting the gastric mucosa, rats were deeply anaesthetized with sodium thiopental $(100 \mathrm{mg} / \mathrm{kg}$ I.P.) and killed by cervical dislocation and exsanguinations. The abdomen was opened; the stomach was excised and cut open along the lesser curvature. Stomachs were stored in Krebs-Henseleit solution until their further use. Gastric content was gently emptied and the mucosa was rinsed with $0.1 \mathrm{M} \mathrm{HCl}$ solution containing $0.9 \%$ sodium chloride $(\mathrm{NaCl})$.

\subsubsection{Detachment force studies}

Detachment force studies were carried out according to the modified surface tensiometer method 21-24. Inner surface of stomach mucosa was outspread on $10 \%$ agaragar gel immobilized with pins. Tablets were fixed with ethyl 2-cyanoacrylate on the bottom of a special specimen hanged on a tensiometer arm (KSV Instruments Ltd, Finland). Before measurements, mucosae were wetted with $20.0 \mu \mathrm{l} 0.1 \mathrm{M} \mathrm{HCl}$ containing $0.9 \% \mathrm{NaCl}$ in order to achieve better mucoadhesive performance. Tablets were left on mucosae surface for 3 minutes.

Maximal detachment forces were recorded and calculated in $\mathrm{mN}$ with the following equation:

Where Fdetach is the detachment force, Ftotal is the measured total weight and Ftablet is the weight of tablet. Structure of measuring method is depicted in (Fig.2). Samples were tested in triplicate.

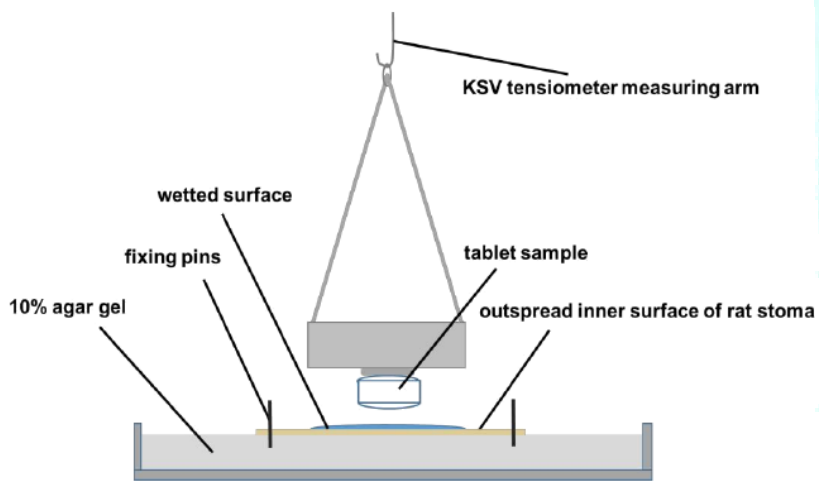

Fig. 2. Structure of tablet detachment force testing apparatus

\subsubsection{Rheological mucoadhesion studies}

Rheological ex vivo mucoadhesion measurements were carried out based on literature 25,26 . Gastric mucus was carefully removed under operating microscope, after which it was put into $0.1 \mathrm{M} \mathrm{HCl}$ containing $0.9 \% \mathrm{NaCl}$. Dispersion with high speed homogenizer (Ultra-Turrax ${ }^{\circledR}$ T 25, IKA ${ }^{\circledR}$, Germany) with 9500 RPM for 2 minutes was performed to achieve homogeneous sample. The mucus then was centrifuged with 5500 RPM for 1 hour. Pellets were dialyzed with Membra-Cel ${ }^{\circledR}$ dialysis tubing (Serva MWCO 3500, Germany) on $4{ }^{\circ} \mathrm{C}$ for 24 hours. Mucus was again centrifuged (Labogene 1524, Denmark) with 15000 RPM for 1 hour. Pellets were stored at $-15{ }^{\circ} \mathrm{C}$ until further use 27,28 .
$3 \%$ mucus solution and optimized formulation equilibrated to $3 \% \mathrm{~L}-\mathrm{HPC}$ and sodium alginate were dispersed separately in $20 \mathrm{ml} 0.1 \mathrm{M} \mathrm{HCl}$. Then mixture of $3 \%$ mucus and MF_OPT equilibrated to $3 \%$ L-HPC and sodium alginate were also prepared.

Flow curves of samples were examined in a rotational viscometer (Anton PaarRheolab QC, Austria) at $37{ }^{\circ} \mathrm{C}$. Data were recorded in a $0-25 \mathrm{~s}^{-1}$ shear rate interval.

Increase of viscosity due to mucoadhesion $(\eta m)$ was calculated with the following formula:

$$
\eta_{m}=\eta_{\text {total }}-\eta_{\text {tabl }}-\eta_{\text {mucus }}
$$

Where, $\eta$ total is the viscosity of mucus/tablet mixture, $\eta$ tabl equilibrated to $3 \% \mathrm{~L}-\mathrm{HPC}$ and sodium alginate and

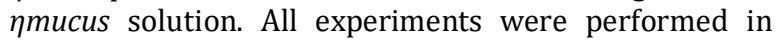
triplicate.

\subsubsection{In vivo $\mathrm{X}$-ray CT evaluation of floating tablets in rat}

Clarithromycin optimized tablets (optimization project) were studied in Wistar rats $(n=5)$. For visualization and detection floating tablets, barium sulfate (BaSO4) X-ray contrast material was used. $10 \%$ of the optimized blend was replaced with BaSO4. The homogenized blend was pressed with $3 \mathrm{~mm}$ round concave punches by eccentric single-punch tablet press.

Before the experiment, rats were kept at room temperature in 12 hour light and dark cycle. The animals were not fasted, food and water were supplied ad libitum. Imaging was performed at the following sampling times: $5 \mathrm{~min}, 1 \mathrm{~h}, 2 \mathrm{~h}, 3 \mathrm{~h}, 4 \mathrm{~h}, 6 \mathrm{~h}, 8 \mathrm{~h}$ and 48 h. Image at 48 hours after administration was examined that floating tablets did not caused gastrointestinal obstruction.

Reconstructed, reoriented and co-registered images were further analyzed by dedicated image analysis software products by placing appropriate volume of interests (VOI) on the tablets. Linear attenuation data were reconstructed into Hounsfield units $(H U)$. Then a second, more detailed, lookup table (LUT) (indicated with different colors) was used by image processing to visualize spectacularly the differences of attenuation values of voxels ordered to the tablets VOIs 29 .

\section{RESULTS AND DISCUSSION}

\subsection{Preformulation methods}

\subsubsection{Comparative physical examination of L-HPC 11 and L-HPC B1}

The photo analysis of L-HPC particles showed differences in shape and in size. (Fig.3). L-HPC 11 had longitudinal shape, while L-HPC B1 formed similar to spheroidal particles. Their shapes were characterized numerically with sphericity index. Sphericity index in the case of L-HPC 11 was $0.19 \pm 0.08$, while in the case of LHPC B1 was $0.48 \pm 0.18$. The result showed that L-HPC B1 is more similar to ideal spherical particles $(\Psi=1.0)$ than L-HPC 11, but its shape is far from sphere shape. 


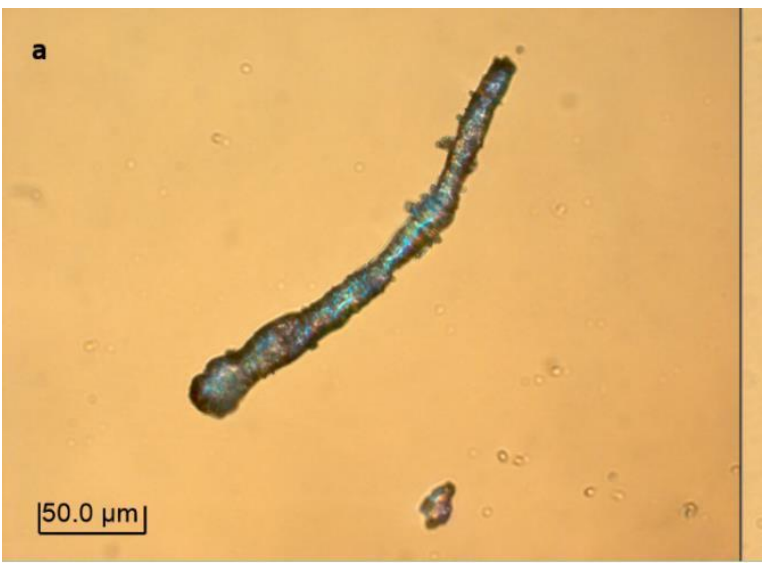

b

Fig. 3. Microscopic appearance of a) L-HPC 11 and b) L-HPC B1 particles

Particle sizes of L-HPC 11 and B1 were also differed. From both materials, 50 particles were evaluated. In the case of L-HPC B1, average size was $44.01 \pm 10.59 \mu \mathrm{m}$ compared to L-HPC 11 with $246.35 \pm 82.03 \mu \mathrm{m}$ average size.

Wettability of $50.0 \mathrm{mg}$ pure L-HPCs were examined in order to reveal further difference between their physicochemical properties. All tests were performed in purified water and in $0.1 \mathrm{M}$ hydrochloric acid for 1 hour, but the powders absorbed most of the fluid in the first 1 minute. Their liquid uptake and Wettability rate as a function of time are shown in figure below.

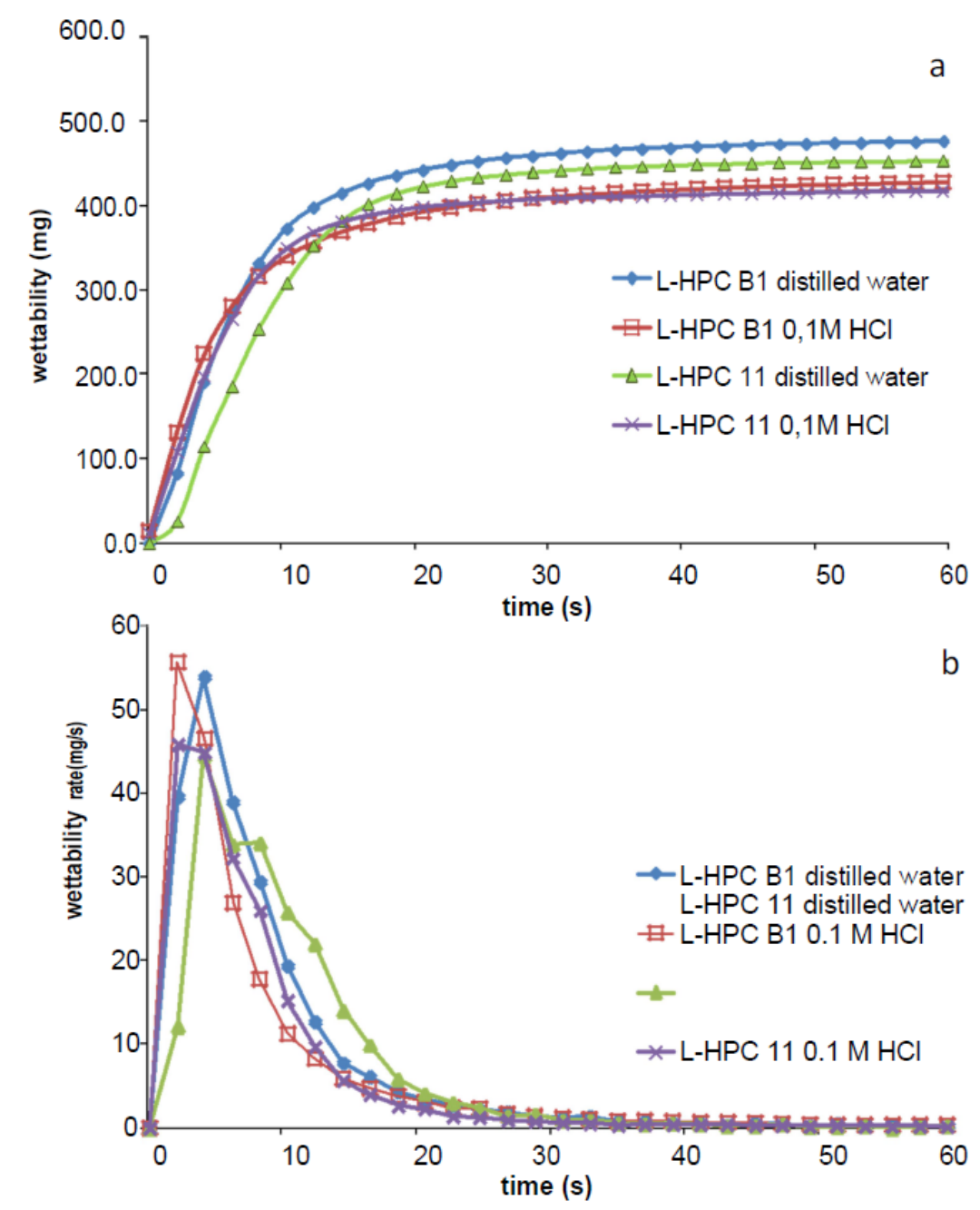

Fig.4. Wettability and wettability rate of L-HPC 11 and B1 as a function of time. 
Final wettability values of examined L-HPCs are shown in Table 3. The result showed that wettability of both materials was lower in $0.1 \mathrm{M} \mathrm{HCl}$ than in distilled water, however, the rate of fluid absorption was higher in $0.1 \mathrm{M} \mathrm{HCl}$. L-HPC B1 showed faster and more intense liquid absorption compared to L-HPC 11.

Table 3. Total wettability of L-HPC 11 and L-HPC B1 after 1 hour

\begin{tabular}{|l|l|l|}
\hline Total wettability of 50 mg L-HPCs & Distilled water (mg) & $0.1 \mathrm{M} \mathrm{HCl} \mathrm{(mg)}$ \\
\hline L-HPC 11 & $483.7 \pm 22.8$ & $451.0 \pm 17.4$ \\
\hline L-HPC B1 & $522.7 \pm 36.6$ & $480.0 \pm 39.6$ \\
\hline
\end{tabular}

Both values (Carr's index and Hausner's ratio) characterizing powder flowability showed better flowability of L-HPC B1. The differences manifested also in tapped $(\rho t)$ and bulk $(\rho b)$ densities.

Table 4. Flow characteristics and densities of L-HPC 11 and L-HPC B1

\begin{tabular}{|l|l|l|l|l|l|}
\hline Samples & $\rho t\left(\mathrm{~g} / \mathrm{cm}^{3}\right)$ & $\rho b\left(\mathrm{~g} / \mathrm{cm}^{3}\right)$ & Carr's index & Hausner's ratio & Angle of repose $\left(^{\circ}\right)$ \\
\hline L-HPC 11 & $0.443 \pm 0.001$ & $0.356 \pm 0.008$ & $22.91 \pm 0.32$ & $1.24 \pm 0.04$ & $48.77 \pm 1.67$ \\
\hline L-HPC B1 & $0.591 \pm 0.003$ & $0.496 \pm 1.012$ & $19.05 \pm 2.31$ & $1.19 \pm 0.02$ & $39.24 \pm 0.86$ \\
\hline
\end{tabular}

Demonstrated data (Table 4) revealed that flowability values differed between L-HPC types, but the differences are not remarkable. The largest deviation was in the case of angle of repose, which can have significant influence during filling the blend into dies.

\subsubsection{Rheological behavior of sodium alginate}

At the preformulation stage of experiments, rheological properties of sodium alginate were determined. High viscosity grade sodium alginate was applied during formulation, which was characterized specifically. The result showed significant dependence of sodium alginate viscosities on temperature. Measured viscosities are presented by (Table 5).

Flow curve measurements showed non-Newtonian, pseudoplastic behavior showing decrease in viscosity caused by increase of shear rate. Calculated flow behavior indices are shown in Table 5, which demonstrates that rheological behavior changes with temperature. Increasing $z$ value results in less change in viscosities caused by increase of shear rate.
Table 5. Temperature dependence of viscosity and flow behavior index of $1 \%$ sodium alginate solutions

\begin{tabular}{|c|c|c|}
\hline$t\left({ }^{\circ} \mathrm{C}\right)$ & $\eta$ (mPas) & $Z$ \\
\hline 20 & $214.73 \pm 7.73$ & $-0,6943$ \\
\hline 25 & $196.88 \pm 7.02$ & $-0,7139$ \\
\hline 30 & $175.65 \pm 7.82$ & $-0,7352$ \\
\hline 35 & $157.12 \pm 6.60$ & $-0,7601$ \\
\hline 37 & $151.63 \pm 6.42$ & $-0,7604$ \\
\hline
\end{tabular}

\subsubsection{Drug-excipients interaction studies}

Result of the thermo analytical method showed a sharp endothermic peak of pure Clarithromycin at $159.9^{\circ} \mathrm{C}$, which was considered as the melting point. Based on the composition of MF_OPT, all the excipients were blended with pure Clarithromycin separately. The samples did not show significant alteration of Clarithromycin melting point, which is between 159 and $163^{\circ} \mathrm{C}$ according to the literature ${ }^{30}$. DTA thermograms of samples are shown in Fig. 5. Consequently, results of this study could not indicate any API-excipient interactions.

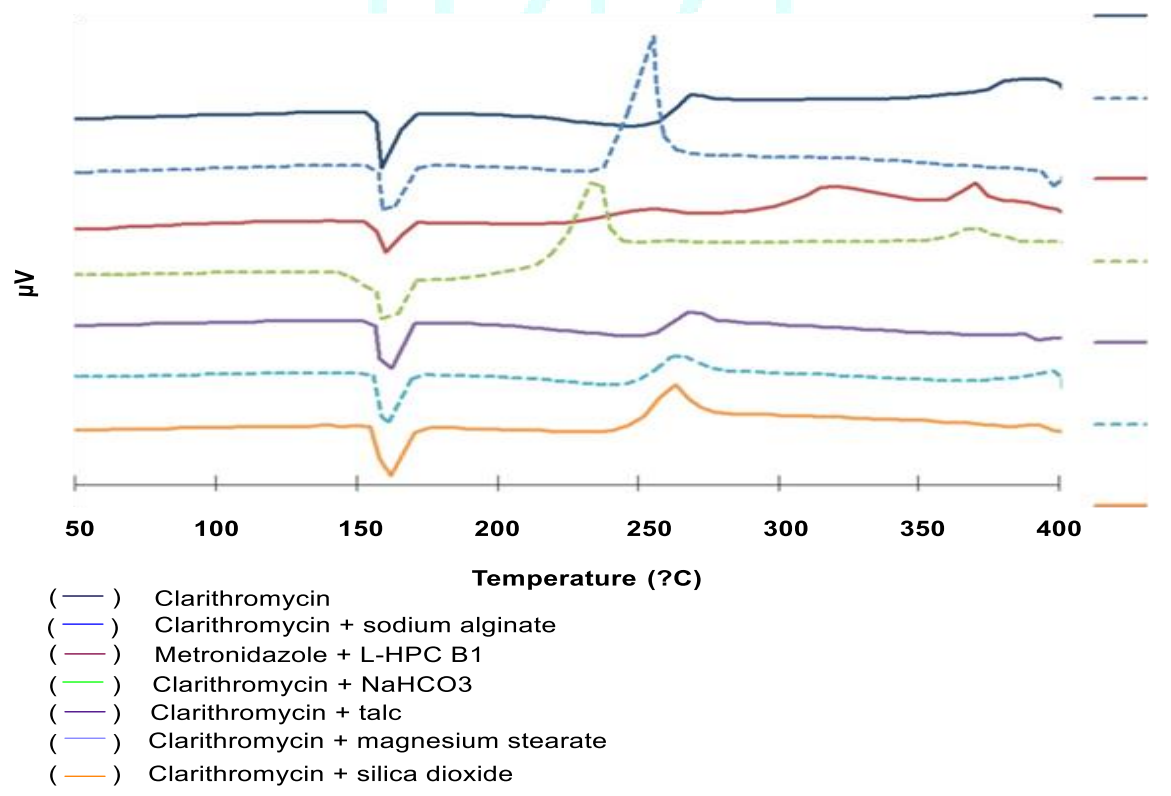

Fig. 5. DTA thermograms of Clarithromycin and Clarithromycin-excipient blends according to MF_OPT composition. 


\subsection{Formulation results}

\subsubsection{Studies of floating behavior}

In the section of floating behavior examinations, floating lag time, total floating time and vertically expressed floating force studies were performed and evaluated.

The best fitting model on floating lag time (tlag) data was the linear model $(p<0.01)$ and sodium alginate was the only significant factor $(p<0.01)$ in the examined concentration ranges. In the lower level $(-1)$ of sodium alginate $(0.5 \%)$ short tlag values were observed, which was $25.77 \pm 6.53 \mathrm{~s}$. Increasing amount of sodium alginate resulted in longer time to achieve buoyancy. At higher sodium alginate levels $(0,+1)$, maximal tlag value was
$520.30 \pm 20.79$ s which can be considered to be long time to float, but only $8 \%$ of sodium bicarbonate accelerated the flotation.

On the total floating time (tfloating) data, quadratic model could be fitted and both numerical factors and their interactions showed significance $(p<0.0001)$. At 0 and +1 levels $(17.82,35.05 \%)$ sodium alginate caused more than 4 hour flotation, since in the cases of tablets having $0.5 \%$ sodium alginate more rapid disintegration could be observed, at which disintegrant effect of L-HPCs could prevail. L-HPC 11 resulted in faster disintegration. Floating lag time and total floating time data are shown in Table 6.

Table 6 Data of floating lag time and total floating time

\begin{tabular}{|l|l|l|l|l|}
\hline Exp. No. & tlag (sec) & Tfloating(min) & Fmax(mN) & tFmax(s) \\
\hline PFS01 & $6.44 \pm 5.19$ & $21.7 \pm 4.6$ & 2.295 & 415.1 \\
\hline PFS02 & $343.00 \pm 28.99$ & $240.0 \pm 0.0$ & 1.549 & 14395 \\
\hline PFS03 & $0.00 \pm 0.00$ & $7.1 \pm 0.5$ & 2.037 & 30.4 \\
\hline PFS04 & $507.16 \pm 100.35$ & $240.0 \pm 0.0$ & 3.884 & 14295 \\
\hline PFS05 & $0.00 \pm 0.00$ & $3.9 \pm 0.3$ & 2.363 & 15.3 \\
\hline PFS06 & $10.16 \pm 75.77$ & $240.0 \pm 0.0$ & 1.904 & 14396 \\
\hline PFS07 & $109.20 \pm 89.32$ & $240.0 \pm 0.0$ & 1.626 & 930 \\
\hline PFS08 & $16.69 \pm 1.58$ & $240.0 \pm 0.0$ & 2.557 & 14398 \\
\hline PFS09 & $520.30 \pm 20.79$ & $240.0 \pm 0.0$ & 1.780 & 13715 \\
\hline PFS10 & $16.02 \pm 6.58$ & $28.6 \pm 1.1$ & 1.375 & 379.2 \\
\hline PFS11 & $356.55 \pm 47.07$ & $240.0 \pm 0.0$ & 2.859 & 14398 \\
\hline PFS12 & $0.00 \pm 0.00$ & $15.5 \pm 3.0$ & 1.292 & 45.6 \\
\hline PFS13 & $230.16 \pm 11.87$ & $240.0 \pm 0.0$ & 4.606 & 3004.8 \\
\hline PFS14 & $0.00 \pm 0.00$ & $15.3 \pm 1.9$ & 1.199 & 141.7 \\
\hline PFS15 & $507.67 \pm 161.30$ & $240.0 \pm 0.0$ & 2.164 & 14386 \\
\hline PFS16 & $273.45 \pm 156.64$ & $240.0 \pm 0.0$ & 0.925 & 14399 \\
\hline PFS17 & $180.00 \pm 51.64$ & $240.0 \pm 0.0$ & 2.677 & 14119 \\
\hline PFS18 & $106.14 \pm 24.18$ & $240.0 \pm 0.0$ & 2.06 & 14389 \\
\hline PFS19 & $25.77 \pm 6.53$ & $35.7 \pm 3.1$ & 1.412 & 475.3 \\
\hline PFS20 & $238.16 \pm 13.25$ & $240.0 \pm 0.0$ & 1.324 & 5450.9 \\
\hline PFS21 & $13.87 \pm 3.25$ & $17.2 \pm 1.3$ & 1.273 & 45.6 \\
\hline PFS22 & $417.22 \pm 18.12$ & $240.0 \pm 0.0$ & 3.270 & 14386 \\
\hline PFS23 & $8.10 \pm 2.62$ & $13.3 \pm 0.3$ & 1.172 & 65.8 \\
\hline PFS24 & $330.33 \pm 27.79$ & $240.0 \pm 0.0$ & 2.315 & 14398 \\
\hline PFS25 & $307.71 \pm 174.70$ & $240.0 \pm 0.0$ & 1.194 & 14398 \\
\hline PFS26 & $344.00 \pm 29.70$ & $240.0 \pm 0.0$ & 2.217 & 14313 \\
\hline PFS27 & $184.00 \pm 24.64$ & $240.0 \pm 0.0$ & 1.899 & 14393 \\
\hline & & & \\
\hline
\end{tabular}

The floating force measurements have been performed for 4 hours. Among samples two patterns could be observed the rapid disintegrating and exponentially increasing patterns. On maximal floating force values (Fmax) data, two-factor interaction model was fitted $(p<0.0001)$.Both numerical factors $(p<0.0001)$ and their interactions $(p=0.0004)$ showed significance. Categorical factor had no significance. On tFmax data, quadratic model was used due to its significant fitting. Time values for achieving maximal floating forces were only influenced significantly by sodium alginate $(p<0.0001)$.

Response curve of tFlag values as a function of L-HPC $11 / \mathrm{B} 1$ mixture, and sodium alginate is depicted by (Fig.6.).

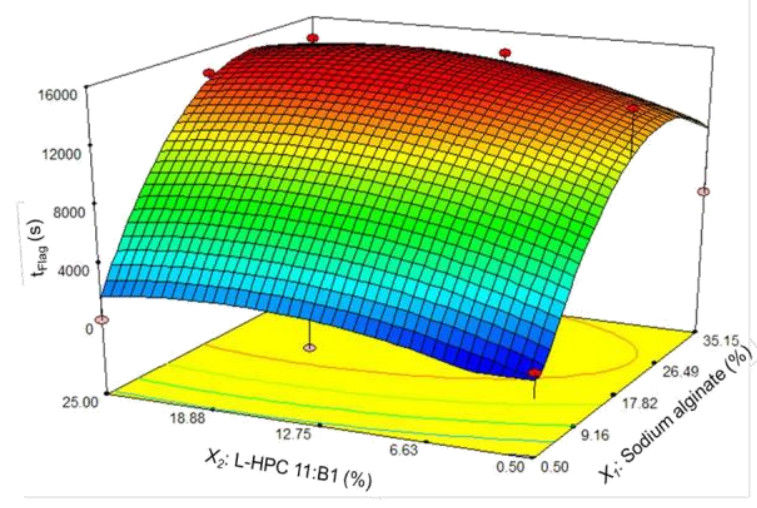

Fig. 6. The effect of L-HPC 11:B1 mixture and sodium alginate on maximal floating force development time 


\subsubsection{Determination of swelling capability}

Swelling capabilities of polymers have one of the most important influences on the behavior in aqueous medium of floating tablets, which are basically matrix tablets. In matrix tablets, during the swelling process, aqueous medium reaches deeper layers of tablets with time, which process is generally driven by diffusion. Drug release of tablets is also affected by swelling, since diffusion creases hydration layers in the tablets resulting in outward diffusion of API. Inside the tablets, relatively dry core with low water content is followed by more hydrated layers, which are surrounded by the surface layer in contact with the medium. The hydration layers of PFS04 floating tablet (L-HPC 11: $25.0 \%$, Sodium alginate: $35.15 \%$ ) can be identified at 4 hour depicted by Fig.7 below.

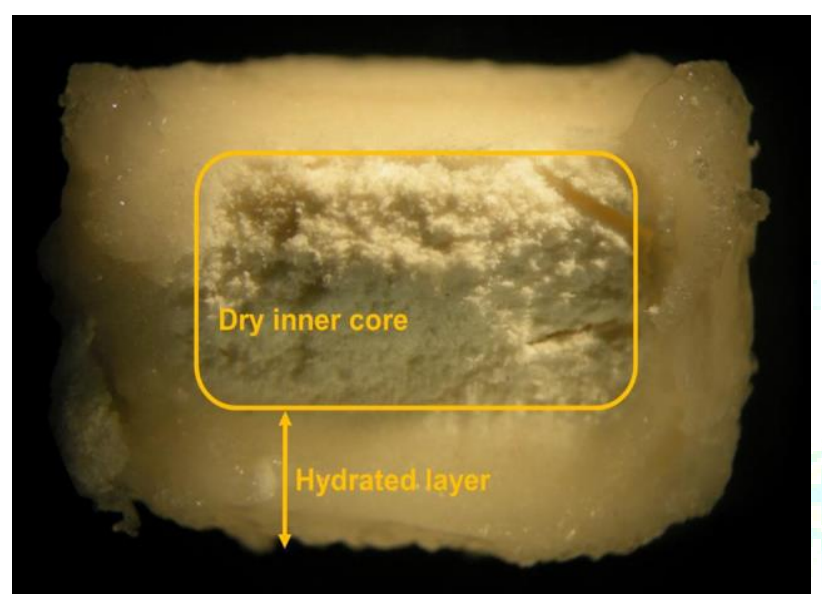

Fig.7. Macroscopic view of PFS04 floating tablets (L-HPC 11: $25.0 \%$, sodium alginate: $35.15 \%$ ) after 4 hours of hydration

Values of $\mathrm{Si}$ indices show the water uptake of tablets, which additionally were standardized for tablet weights. On the swelling capability data, linear model could be fitted with high significance $(p<0.01)$. At $S i$ values after 30 minutes and 1 hour, sodium alginate was the only significant factor $(p<0.01)$, but at 2 hours L-HPC $(X 2)$ was also significant $(p<0.05)$. The categorical factor was not significant. Maximal swelling index ( $\mathrm{Si}=2.357 \pm 0.067)$ was observed at PFS22 (L-HPC 11/B1: $25.0 \%$, sodium alginate: $35.15 \%$ ), which is significantly higher, than published $S i$ values of sodium alginate based floating tablets ${ }^{31}$.

\subsubsection{Paracetamol release studies}

Dissolution studies of paracetamol were carried out for 4 hours. In this time interval, various release profiles could be observed but two main types could be distinguished and identified: rapid disintegrating - and prolonged drug release.

Those tablets in which sodium alginate concentration was in lower level (-1: $0.5 \%)$ released the API fast. Floating tablets with more than $17.82 \%$ sodium alginate showed sustained release. This phenomenon may be due to that $0.5 \%$ sodium alginate concentration was not enough to create coherent structure, thus the effect of LHPCs could manifest. On the other hand, floating tablets with sustained release could produce maximally $32.99 \pm 3.40 \%$ dissolution after 4 hours. In the analysis of variance (ANOVA), two sections could be identified based on significance of factors. All dissolution data were fitted with quadratic model $(p<0.0001)$. In the first time period (from 5 to $45 \mathrm{~min}$ ), sodium alginate $(X 1)$, L-HPC $(X 2)$ and their interaction $(X 1 X 2)$ were significant $(p<0.05)$. At $45 \mathrm{~min}$, categorical factor $(X 3)$ became significant, too. In the second period (from 1 to 4 hours), sodium alginate and the categorical factor were only significant $(p<0.05)$, which indicated that the influence of L-HPC was not permanently significant on dissolution only until 45 minutes. Difference between L-HPC types could only be observed in this period.

\subsubsection{Clarithromycin release studies}

In vitro dissolution studies of all floating tablets were performed for 6 hours and its result are depicted in (Fig.8).

The most rapid dissolution could be observed at MF07 (sodium alginate: $5.0 \%$, L-HPC B1: $45.0 \%$, sodium bicarbonate: $13.0 \%$ ) having total dissolution after 60 minutes. Compositions with more than $5.0 \%$ sodium alginate $(10.0$ or $15.0 \%)$ could not produce more than $26.87 \pm 1.05 \%$ dissolution after 6 hours. In the case of 5.0 $\%$ sodium alginate, the least released amount of Clarithromycin was 80-82 \% (MF01, MF05).

Standard deviations (SD) of all dissolution data were also analyzed with ANOVA and sodium alginate was found as a significant factor influencing SD values $(p<0.05)$. The more sodium alginate quantity the tablets had the less SD values were observed, which consequently could influence manufacturing reproducibility. 

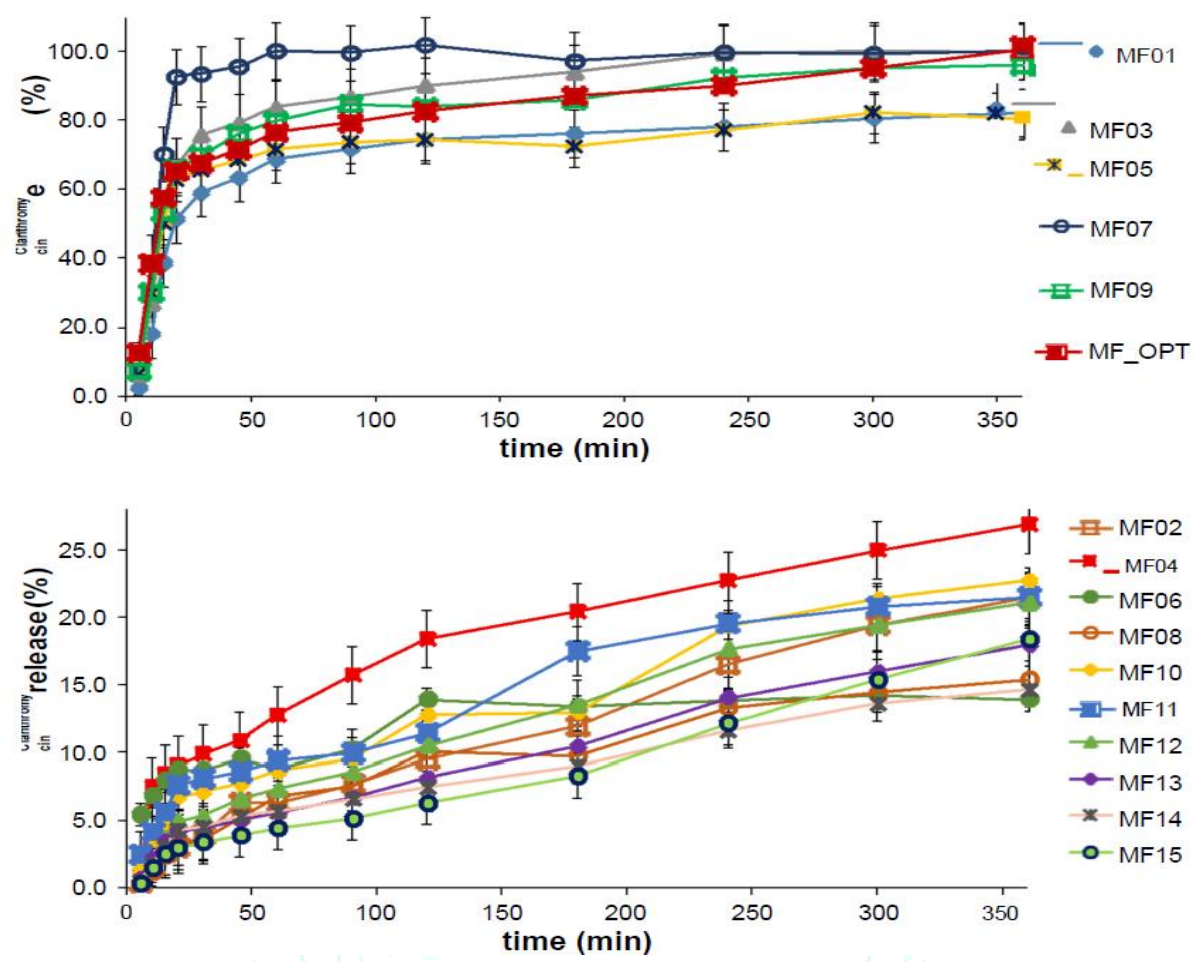

Fig.8. Dissolution profiles of floating tablets (Optimization Project)

\subsubsection{Drug release kinetics}

Evaluation of Clarithromycin release revealed that tablets having sustained dissolution (MF2, MF4, MF6, MF8, MF10, MF11, MF12, MF13, MF14, MF15) can be characterized by Higuchi model, which refers to drug diffusion from polymer matrices. These floating tablets contained 10.0 or $15.0 \%$ sodium alginate. In the case of low sodium alginate concentration (5.0\%), none of the applied models fitted significantly. Result of kinetics analysis is shown in Table 10. The best fitted release model of MF_OPT was first order kinetics, but due to the biphasic dissolution coefficient of determination was only $R^{2}=0.820$.

Table 7. Result of model dependent evaluation of drug release (optimization project)

Zero order model First order model Higuchi model

Weibull model

\begin{tabular}{|c|c|c|c|c|c|c|c|c|c|c|c|c|}
\hline Sample & $m$ & $n$ & $R^{2}$ & $m$ & $n$ & $2 \quad R 2$ & $m$ & $n$ & $\begin{array}{l}2 \\
R 2 \\
\end{array}$ & $m$ & $n$ & \begin{tabular}{|l|} 
\\
$R 2$ \\
\end{tabular} \\
\hline MF1 & 0.23 & 37.64 & 0.509 & -0.05 & 4.08 & 0.678 & 4.81 & 18.53 & 0.693 & 0.83 & -3.54 & 0.728 \\
\hline MF2 & 0.06 & 1.82 & 0.964 & -0.00 & 4.59 & 0.969 & 1.09 & -1.92 & 0.980 & 0.82 & -6.12 & 0.939 \\
\hline MF3 & 0.28 & 48.88 & 0.518 & -0.02 & 4.20 & 0.896 & 5.65 & 26.63 & 0.693 & 0.90 & -3.20 & 0.860 \\
\hline MF4 & 0.08 & 6.75 & 0.857 & 0.00 & 4.54 & 0.883 & 1.43 & 1.64 & 0.944 & 0.63 & -4.58 & 0.758 \\
\hline MF5 & 0.18 & 46.33 & 0.373 & 0.00 & 3.91 & 0.500 & 3.77 & 30.87 & 0.546 & 0.60 & -2.44 & 0.664 \\
\hline MF6 & 0.03 & 7.34 & 0.808 & 0.00 & 4.53 & 0.812 & 0.61 & 5.15 & 0.888 & 0.24 & -3.20 & 0.917 \\
\hline MF7 & 2.00 & 24.71 & 0.658 & -0.08 & 4.55 & 0.817 & 19.96 & -19.83 & 0.789 & 1.72 & -4.82 & 0.891 \\
\hline MF8 & 0.05 & 2.03 & 0.900 & 0.00 & 4.59 & 0.909 & 0.93 & -1.26 & 0.972 & 0.84 & -6.32 & 0.919 \\
\hline MF9 & 0.24 & 48.80 & 0.481 & 0.00 & 3.89 & 0.771 & 4.92 & 29.24 & 0.657 & 0.72 & -2.66 & 0.785 \\
\hline MF10 & 0.06 & 3.99 & 0.911 & 0.00 & 4.57 & 0.919 & 1.12 & 0.13 & 0.940 & 0.58 & -4.76 & 0.906 \\
\hline MF11 & 0.07 & 4.69 & 0.934 & 0.00 & 4.56 & 0.942 & 1.16 & 0.75 & 0.946 & 0.49 & -4.26 & 0.942 \\
\hline MF12 & 0.06 & 2.64 & 0.954 & 0.00 & 4.58 & 0.963 & 1.15 & -1.28 & 0.977 & 0.75 & -5.66 & 0.900 \\
\hline MF13 & 0.05 & 2.36 & 0.958 & 0.00 & 4.58 & 0.963 & 0.86 & -0.55 & 0.962 & 0.63 & -5.35 & 0.909 \\
\hline MF14 & 0.04 & 2.53 & 0.897 & 0.00 & 4.58 & 0.907 & 0.72 & 0.00 & 0.959 & 0.67 & -5.63 & 0.858 \\
\hline MF15 & 0.04 & 1.53 & 0.960 & 0.00 & 4.59 & 0.961 & 0.74 & -0.96 & 0.946 & 0.72 & -6.03 & 0.887 \\
\hline MF_OPT & 0.22 & 50.30 & 0.524 & 0.00 & 3.89 & 0.820 & 4.40 & 33.06 & 0.690 & 0.59 & -2.13 & 0.816 \\
\hline Klio $^{\circledR}{ }^{\circledR}$ & 0.56 & 51.16 & 0.497 & -0.04 & 3.59 & 0.618 & 8.66 & 23.56 & 0.671 & 1.07 & -3.07 & 0.866 \\
\hline $\operatorname{Suppli}_{\mathrm{n}}^{\circledR}$ & 6.07 & -7.26 & 0.833 & -0.34 & 6.24 & 0.897 & 42.31 & -76.73 & 0.899 & 3.07 & -7.23 & 0.953 \\
\hline
\end{tabular}




\subsubsection{Ex-vivo mucoadhesion studies}

Mucoadhesion is one of the approaches of gastroretentive systems, which can be to combine with other gastroretentive technologies. Their combination may result in more prolonged gastric retention, with which location of drug release can be more predictable. This synergic effect is more desirable for drug delivery systems with APIs aimed at the site of gastric mucosa. In this optimization project, the aim was to design and develop a floating tablet containing Clarithromycin for Helicobacter pylori eradication from gastric mucosa.

In the ex vivo mucoadhesive studies, the two most frequently performed mucoadhesion measurements were done in order to present the potential in mucoadhesive properties of MF_OPT tablets: detachment force and rheological mucoadhesion studies.

Ex vivo mucoadhesion studies evaluated the mucoadhesion of floating tablets in different ways. Detachment force study evaluates the possible mucoadhesion in a relatively dry status. Mucoadhesion is based on the dehydration theory ${ }^{32}$. In contrast with the rheological method, this indirectly measures mucoadhesion based on macromolecular interpenetration ${ }^{33}$. Rheological method interprets the viscosity changes, when the tablet components are in dissolved and swollen form.

Detachment force studies were carried with MF_OPT and three other tablets with modified composition as reference having sodium alginate, L-HPC B1 (MF_OPT_LHPC) or both excipient (MF_OPT_EXC) absences from composition. The result of detachment force study is shown in Fig. 9. MF_OPT tablets have resulted in remarkably higher detachment force $(505.49 \pm 45.62 \mathrm{mN})$ compared to MF_OPT_L-HPC $(314.91 \pm 37.88 \mathrm{mN})$ and MF_OPT_EXC $(264.68 \pm 15.42 \mathrm{mN})$. Tablets without L-HPC B1 had higher detachment force than the reference without both excipients. This study may show the potential in the possible physical synergistic effect between the applied gel forming polymer and disintegrant affected with rapid water absorption.

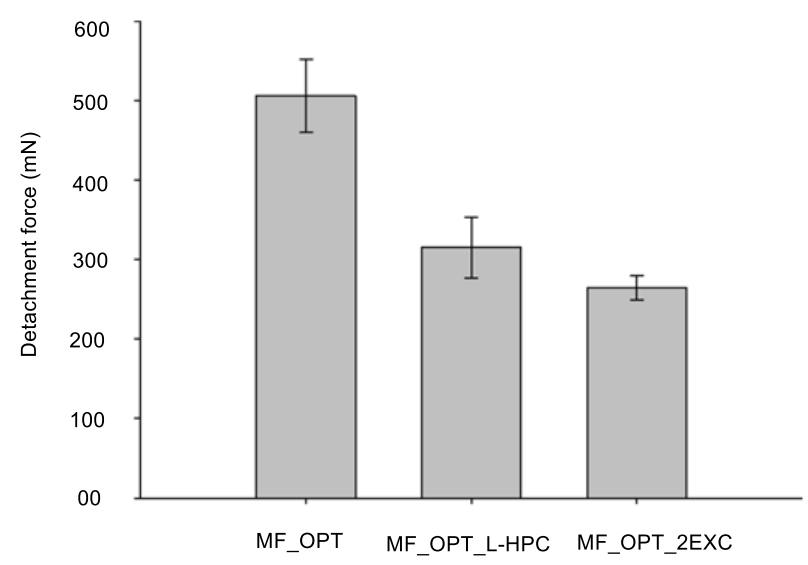

Fig. 9. Result of the detachment force study of MF_OPT (5.0 \% sodium alginate, $38.63 \%$ HPC B1, $8.45 \%$ sodium bicarbonate) and reference tablets without sodium alginate and/ or L-HPC B1 (MF_OPT - optimized composition, MF_OPT_L-HPC - MF_OPT without L- HPC B1, _OPT_2EXC - MF_OPT without sodium alginate and L_HPC)

Result of MF_OPT tablets with the absence of sodium alginate led to splitting of tablets due to rapid hydration effect of L-HPC B1. Less coherence of the tablet structure was observed at tablets without sodium alginate in the presence of acidic medium, therefore these tablets were not evaluated. Low viscosities were measured at $3 \%$ mucus $(7.63 \pm 1.24 \mathrm{mPas})$ and at MF_OPT tablet dispersion $(27.57 \pm 23.22 \mathrm{mPas})$. Mixture of tablet and mucus showed significant rise of viscosity (846.89 $\pm 78.25 \mathrm{mPas}$ at $2.631 / \mathrm{s})$. At low shear rates having the greatest interest 34 , eightfold increase could be observed. Flow curve of mixture of tablet and mucus showed plastic flow behavior. Result of rheological method is shown in (Fig. 10.)

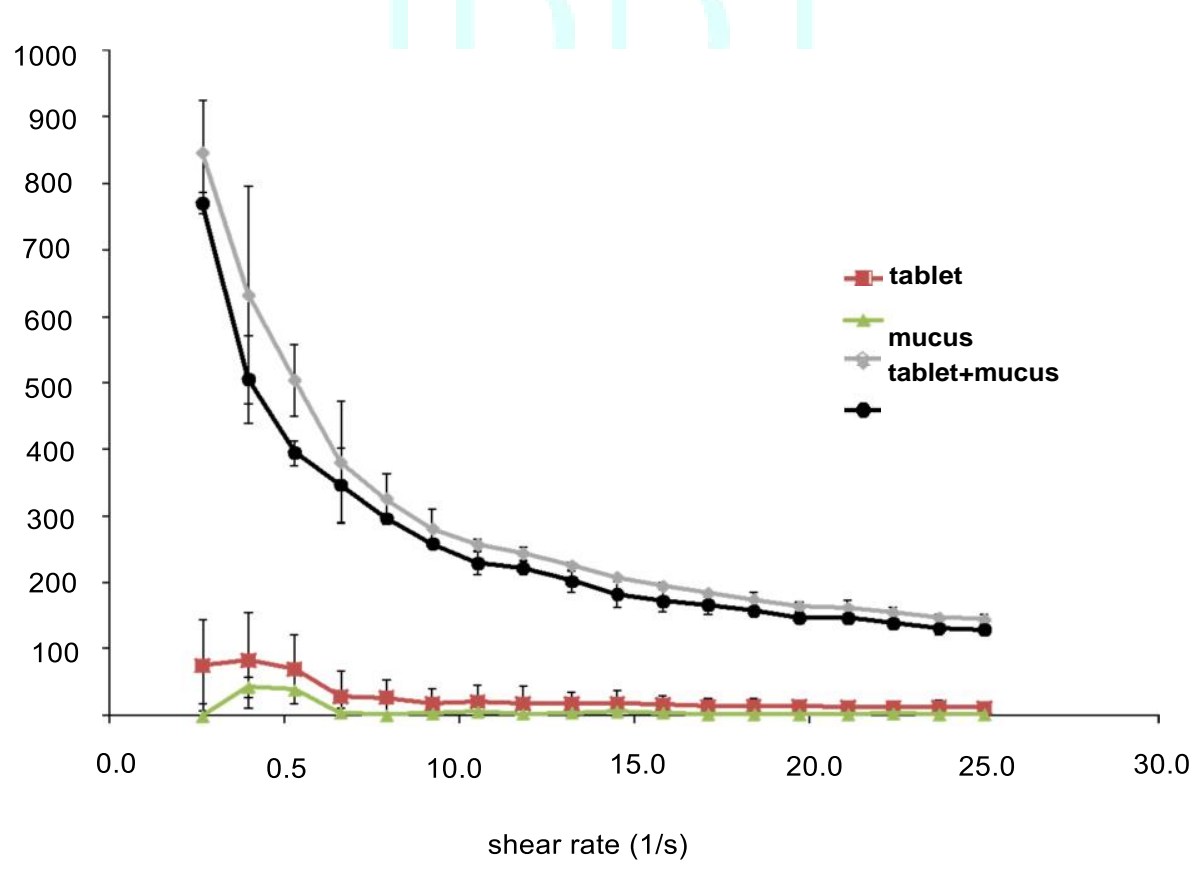

Fig.10. Result of ex vivo rheological mucoadhesion studies of $3 \%$ mucus, MF_OPT equilibrated to $3 \%$ L-HPC and sodium alginate (tablet), their mixture (tablet + mucus) and calculated viscosity increase signed as 'mucoadhesion' 


\subsubsection{In vivo X-ray CT evaluation of floating tablets in rat}

In vivo gastric retention of MF_OPT tablets with $10 \%$ BaSO4 were examined using rat model, which was performed as a correlation to human model in a costeffective way in order to gain valuable preliminary information. $H U$ values of tablets at sampling times showed (Table 8) that $10 \%$ BaSO 4 content in tablets may result in significantly $(\mathrm{p}<0.0001)$ higher $H U$ value in comparison to the liver's $H U$ as reference (HUliver = 800). Thus, tablets could be distinguished from tissues interfering with the view of the tablets.

Fig.11. showed the fact that MF_OPT tablets could remain in stomach for at least 8 hours. The period of gastric retention could be enough regarding the fact that more than $96 \%$ of API was released within 6 hours based on the results of in vitro spectrophotometric and microbiologically detected dissolution studies. After 48 hours, tablets could be identified in the intestinal tract. The prolonged residence time in intestinal region may be due to the effect of anesthesia, which effect is published by Torjman et al. 35 .

Table.8. Mean Hounsfield Units and quantities of voxels inside VOIs of MF_OPT tablets with $10 \%$ BaSO4 at sampling times

\begin{tabular}{|c|c|c|}
\hline $\mathrm{t}$ & Mean Hounsfield unit $(H U)$ & $\begin{array}{c}\text { Quantities of } \\
\text { voxels }\end{array}$ \\
\hline $5 \mathrm{~min}$ & $1482.35 \pm 304.03$ & 1735 \\
\hline $1 \mathrm{~h}$ & $1165.00 \pm 103.23$ & 1132 \\
\hline $2 \mathrm{~h}$ & $1152.38 \pm 106.90$ & 1755 \\
\hline $3 \mathrm{~h}$ & $1202.83 \pm 155.36$ & 1949 \\
\hline $4 \mathrm{~h}$ & $1194.20 \pm 146.70$ & 1815 \\
\hline $6 \mathrm{~h}$ & $1151.43 \pm 104.99$ & 1341 \\
\hline $8 \mathrm{~h}$ & $1151.27 \pm 106.23$ & 1324 \\
\hline $48 \mathrm{~h}$ & $1197.29 \pm 112.15$ & 870 \\
\hline
\end{tabular}

(a)
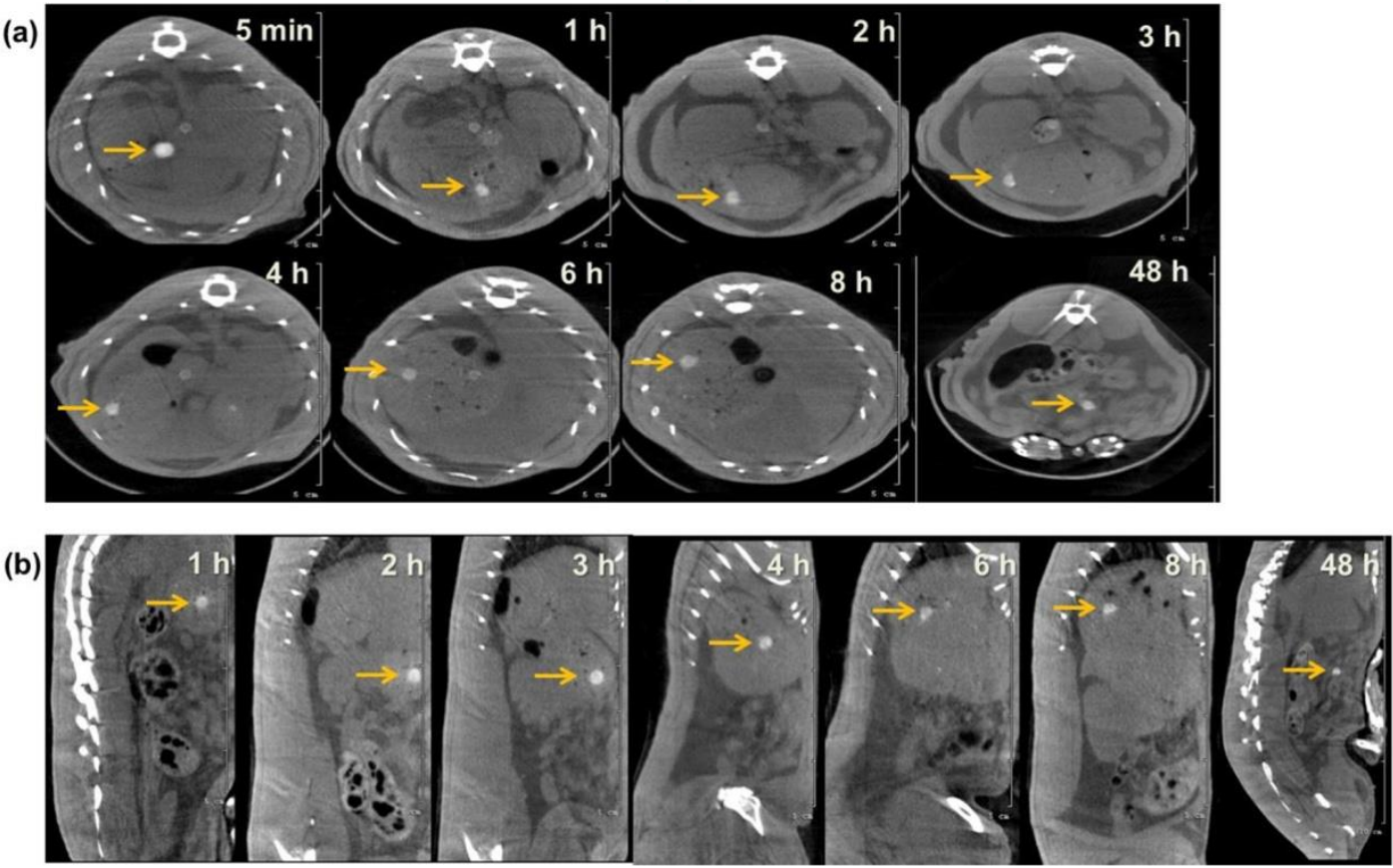

Fig. 11. X-Ray CT images of MF_OPT tablets loaded with $10 \%$ barium sulfate at different time periods in transverse (a) and in sagittal plane (b) (location of tablets indicated with arrows)

The position of MF_OPT tablet in gastrointestinal tract at 8 hours is shown with yellow in (Fig. 12) and (Fig. 13). The homogeneity parameter of the tablet could be visualized in a spectacular manner. In the Fig. 11/A, identifications of voxels were performed in wide range, with which the location of tablets could be visualized. In (Fig. 11/B), tablet structure may be viewed by the
Imaging of the dispersion of $\mathrm{BaSO} 4$ particles. This fine resolution X-Ray CT image visualized the voxels for the VOIs of tablet (indicated with yellow colors) and for VOIs of background (a conventional grey scale). With the application of this technique, valuable information could be gained related to the in situ behavior of tablets including disintegration, swelling, gas creation etc. 

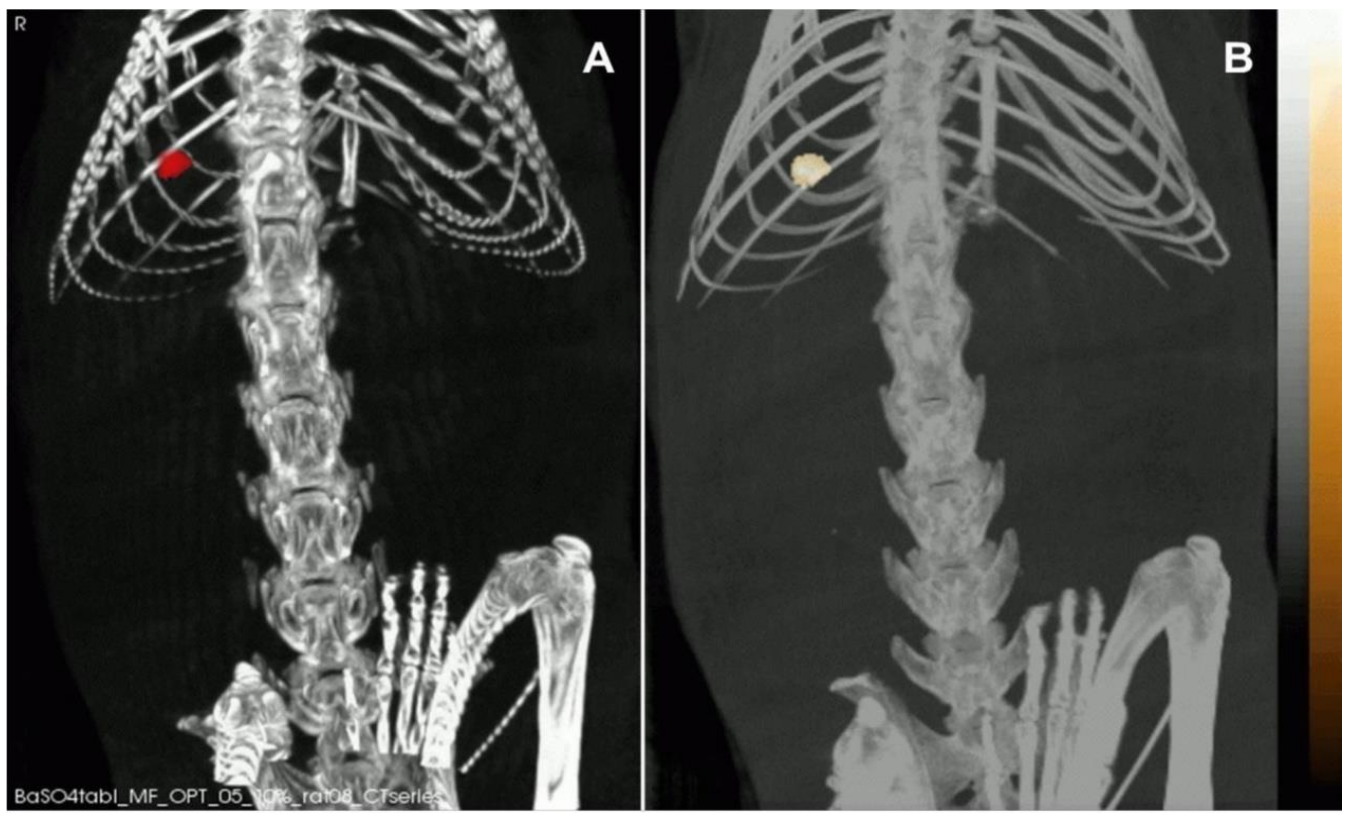

Fig.12. Position of MF_OFT tablets in rat at 8 hours A: Identification of tablets with simple X-Ray CT evaluation; B: Identification and quantification of tablets with fine resolution X- Ray CT technique applying two different lookup tables

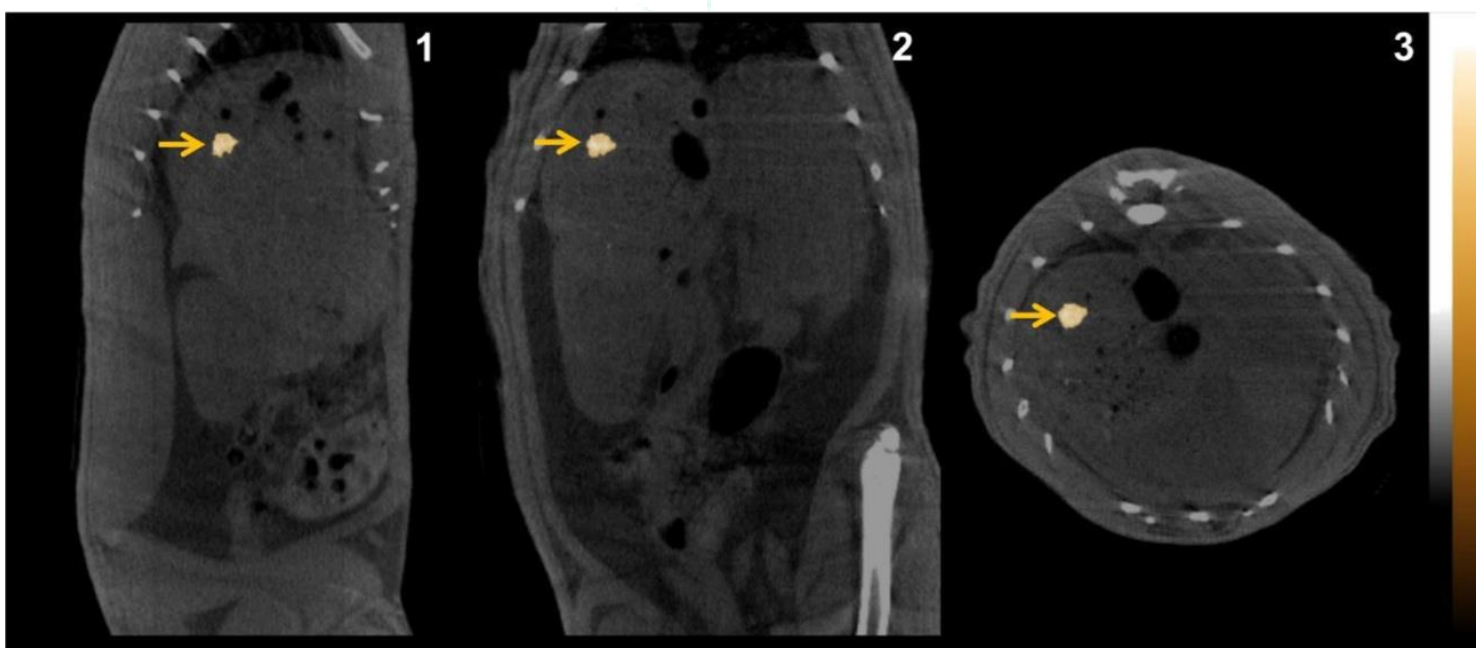

Fig.13. In vivo images of the position and structure of MF_OPT tablet at 8 hour after administration in different planes (1 axial, 2 - coronal, 3 - sagittal)

\subsection{Optimization of technological and biopharmaceutical parameters}

In order to determine a composition of a desired floating tablet, optimization was carried out based on the evaluation of floating behavior and release experimental parameters. One of the aims was to minimize amount of excipients, since less excipients amount are applied, the more active substance(s) can be loaded into tablets. Floating parameters were adjusted so that the formulation would have the best floating properties within this concentration interval manifesting in minimization of tlag and maximization of Fmax and Fmax/100mg. The proportion of release until the first 30 minutes was minimized, after 30 minutes it was maximized.

Based on the optimization criteria, an optimal composition (MF_OPT) was determined having $5.0 \%$ sodium alginate, $38.63 \%$ L-HPC B1 and $8.45 \%$ sodium bicarbonate by Design Expert 7.0.0 software. Floating parameters were also ascertained. Dissolutions of two commercially available non-floating Clarithromycin generics were tested, in order to compare them with the optimized formulation.
Results showed that dissolution of MF_OPT could be regarded to be biphasic release involving an initiative rapid - $(\sim 60 \%)$ and a prolonged release section $(\sim 40 \%)$. MF_OPT tablets had several advantages including a biphasic release and remarkable floating parameters compared to the rapidly released Clarithromycin from the two approved tablets.

\section{CONCLUSION}

The floating-, swelling behavior and dissolution of the preliminary floating tablets were highly affected by the amount of sodium alginate (X1) in the compositions. L-HPC resulted in rapid disintegration, but this effect could only be manifested, when lower quantity of the matrix former (sodium alginate in $0.5 \%$ ) was present, when sodium alginate was applied in 17.82 and $35.05 \%$, then high swelling capability and sustained dissolution could be observed as well as longer floating lag time and floating time, L-HPC as numerical factor $(X 2)$ was significant in several cases, but its effect was less than the effect of sodium alginate concentration (in this concentration range), categorical factor (X3) was only significant at 
dissolution after $45 \mathrm{~min}$, hence the significance could be identified but the difference was not remarkable, therefore for further studies L-HPC B1 was used considering its better flowability properties Floating lag time data indicated that $8 \%$ sodium bicarbonate may have to be increased in order to achieve faster start of buoyancy, the range of L-HPC and sodium alginate concentrations was too broad, thus further adjustment in this interval was required in order to create a floating drug delivery system with desirable floating, swelling, dissolution properties. Clarithromycin dissolution kinetic results showed various release behavior. Trend could be observed that best fitting model of releases from floating tablets with 10.0 or $15.0 \%$ sodium alginate was Higuchi model. Optimization of factors could be done by the use of optimization criteria. The optimized composition contained $5.0 \%$ sodium alginate $(X 1), 38.63 \%$ L-HPC B1 (X2), $8.45 \%$ sodium bicarbonate $(X 3)$. The optimized formulation showed promising properties including low floating lag time $($ tlag $=13.25 \pm 0.50 \mathrm{~s})$, high floating force $(F \max =12.75 \pm 1.87$ $\mathrm{mN}$ ) and biphasic drug dissolution. Between drug and excipients, studies did not reveal any interactions. X-Ray CT imaging result showed a prolonged in vivo retention of floating tablets in gastric region.

\section{REFERENCES}

1. A. Dévay, I. Antal, A gyógyszeres terapia biofarmáciai alapjai, Medicina Könyvkiadó (2009).

2. N. Narang, An updated review on: Floating drug delivery system, Int J Appl Pharm,3 (2010) 1-7.

3. Y.H. Bae, K. Park, Targeted drug delivery to tumors: myths, reality and possibility, J Control Release, 153 (2011) 198205.

4. E.A. Klusner, E. Lavy, M. Friedman, A. Hoffman, Expandable gasrtroretentive dosage forms, J Control Release, 2 (2003) 143-162. .

5. E.A. Klusner, E. Lavy, D. Stepensley, M. Friedman, A Hoffman, Novel gasrtroretentive dosage form: evaluation of gastroretentivity and its effect on riboflavin absorption in dogs, Pharm Res, 19 (2002) 1516-1523.

6. G.M. Clarke, J.M. Newton, M.D. Short, Gastrointestinal transit of pellets of differing size and density, Int J Pharm, 100 (1993) 81-92.

7. Y. Huang, W. Leobandung, A. Foss, N.A. Peppas, Molecular aspects of muco- and bioadhesion: tethered structures and site-specific surfaces, J Control Release, 65 (2000) 63-71.

8. USP, 2007. Powder flow <1174> USP30 NF 25.

9. R. Carr, Evaluating flow properties of solids, Chem Eng, 72 (1965) 163-169.

10. D.V. Boger, A.L. Halmos, Characterization of fluid behaviour (Module C 2.1), in: Non Newtonian flow, Am Inst Chem Eng J, 1981.

11. J. Timmermans, A.J. Moes, Measuring the resultant-weight of an immersed test material. 1. Validation of an apparatus and a method dedicated to pharmaceutical applications, Acta Pharm Technol, 36 (1990) 171-175.

12. J. Timmermans, A.J. Moës, Determining in vitro the resultant-force acting on a pharmaceutical form immersed in a fluid, an apparatus and a method, Proceedings of the Fifth APGI International Conference on Pharmaceutical Technology Part 2, (1989) 294-303.

13. A.H. Cromer, Physics for life sciences. 2 nd Ed. Int. Student Edition McGraw-Hill Intern. Book co. Tokyo, Japan, (1981) 134-153.

14. P. Dorozynski, R. Jachowicz, P. Kulinowski, S. Kwiecinski, K. Szybinski, T. Skorka, A. Jasinski, The macromolecular polymers for the preparation of hydrodynamically balanced systems-methods of evaluation, Drug Dev Ind Pharm, 30 (2004) 947-957.

15. P. Costa, J.M. Sousa Lobo, Modeling and comparison of dissolution profiles, Eur J Pharm Sci, 13 (2001) 123-133.
16. L. Bruner, S. Tolloczko, Über die Auflösungsgeschwindigkeit Fester Körper, Z Phys Chem, (1901) 283-290.

17. N.V. Mulye, S.J. Turco, A simple model based on first order kinetics to explain release of highly water soluble drugs from porous dicalcium phosphate dihydrate matrices, Drug Dev Ind Pharm, 21 (1995) 943-953.

18. T. Higuchi, Mechanism of sustained-action medication. Theoretical analysis of rate of release of solid drugs dispersed in solid matrices, J Pharm Sci, 52 (1963) 11451149.

19. A. Devay, P. Fekete, I. Racz, Application of factorial design in the examination of Tofizopam microcapsules, J Microencapsul, 1 (1984) 299-305.

20. E. Nagy, U.S. Justesen, Z. Eitel, E. Urban, E.S.G.A. Infecti, Development of EUCAST disk diffusion method for susceptibility testing of the Bacteroides fragilis group isolates, Anaerobe, 31 (2015) 65-71.

21. J.D. Smart, I.W. Kellaway, H.E. Worthington, An in vitro investigation of mucosa adhesive materials for use in controlled drug delivery, J Pharm Pharmacol, 36 (1984) 295-299.

22. H. Park, J.R. Robinson, Physico-chemical properties of water insoluble polymers important to mucin/epithelial adhesion, J Control Release, 2 (1985) 47-57.

23. D.E. Chickering, E. Mathiowitz, Bioadhesive Microspheres .1. A novel electrobalance based method to study adhesive interactions between individual microspheres and intestinal mucosa, J Control Release, 34 (1995) 251-262.

24. J.D. Smart, An Invitro assessment of some mucosa-adhesive dosage forms, Int J Pharm, 73 (1991) 69-74.

25. D. Ivarsson, M. Wahlgren, Comparison of in vitro methods of measuring mucoadhesion: Ellipsometry, tensile strength and rheological measurements, Colloid Surf B, 92 (2012) 353-359.

26. E.E. Hassan, J.M. Gallo, A simple rheological method for the in vitro assessment of mucin-polymer bioadhesive bond strength, Pharm Res, 7 (1990) 491-495.

27. F. Madsen, K. Eberth, J.D. Smart, A rheological examination of the mucoadhesive/mucus interaction: the effect of mucoadhesive type and concentration, J Control Release, 50 (1998) 167-178.

28. C.M. Lehr, H.E. Bodde, J.A. Bouwstra, H.E. Junginger, A surface-energy analysis of mucoadhesion. 2. Prediction of mucoadhesive performance by spreading coefficients, Eur J Pharm Sci, 1 (1993) 19-30.

29. M. M. Balda, D. Niederlohner, B. Kreisler, J. Durst, H. B., Lookup table-based simulation of directly-converting counting X-ray detectors for computed tomography, Nuclear Science Symposium Conference Record (NSS/MIC), IEEE, (2009) 2588-2593.

30. E.G.C. Clarke, Clarke's Isolation and Identification of Drugs: In Pharmaceuticals, in: Body fluids and post mortem material, The Pharmacutical Press, London, (1986) 780781.

31. X. Qi, H. Chen, Y. Rui, F. Yang, N. Ma, Z. Wu, Floating tablets for controlled release of ofloxacin via compression coating of hydroxypropyl cellulose combined with effervescent agent, Int J Pharm, 489 (2015) 210-217.

32. J.D. Smart, The role of water movement and polymer hydration in mucoadhesion, in: Bioadhesive Drug Delivery Systems: Fundamentals, Novel Approaches and Development, Marcel Decker, (1999) 11-23.

33. E. Jabbari, N. Wisniewski, N.A. Peppas, Evidence of mucoadhesion by chain interpenetration at a poly(acrylic acid)/mucin interface using ATR-FTIR spectroscopy, J Control Release, 27 (1993) 89-99.

34. D. Ivarsson, M. Wahlgren, Comparison of in vitro methods of measuring mucoadhesion: Ellipsometry, tensile strength and rheological measurements, Colloid Surf B, 92 (2012) 353-359.

35. M.C. Torjman, J.I. Joseph, C. Munsick, M. Morishita, Z. Grunwald, Effects of Isoflurane on gastrointestinal motility after brief exposure in rats, Int J Pharm, 294 (2005) 65-71. 